\title{
Prognostic value of mean platelet volume/platelet count ratio in patients with resectable esophageal squamous cell carcinoma: a retrospective study
}

\author{
Ji-Feng Feng ${ }^{\text {Corresp., } 1,2}$, Chen Sheng ${ }^{1}$, Qiang Zhao ${ }^{1,2}$, Peng-Cheng Chen ${ }^{\text {Corresp. } 1}$ \\ 1 Department of Thoracic Surgery, Zhejiang Cancer Hospital, Hangzhou, China \\ 2 Key Laboratory Diagnosis and Treatment Technology on Thoracic Oncology, Hangzhou, China \\ Corresponding Authors: Ji-Feng Feng, Peng-Cheng Chen \\ Email address: Fengjf@zjcc.org.cn, Chenpc0425@126.com
}

Background. Mean platelet volume (MPV) to platelet count (PC) ratio (MPV/PC) is a useful indicator in several cancers. However, the role for MPV/PC ratio in esophageal squamous cell carcinoma (ESCC) is still controversial.

Methods. A retrospective study was conducted including 277 resectable ESCC patients. The optimal cutoff values were calculated by the X-tile program. The receiver operator characteristic (ROC) curves were also created to show the candidate cut-off points. The comparisons between the X-tile plot and ROC curve were performed. The Kaplan-Meier method was utilized to analyze the cancer-specific survival (CSS). Prognostic factors for CSS were calculated with Cox regression univariate and multivariate analyses.

Results. According to the X-tile program, the cut-off values for MPV, PC and MPV/PC ratio were 8.5 (fl), 200 (giga/l) and 0.04, respectively. However, the cut-off values for MPV, PC and MPV/PC ratio by the ROC curves were 8.25 (fl), 243.5 (giga/l) and 0.0410, respectively. The cut-off values were similar between the X-tile and ROC curve. A low MPV/PC ratio level ( $\leq 0.04)$ was associated with poor CSS $(22.4 \%$ vs. $43.1 \%, P$ $<0.001$ ). In multivariate analyses, we found that MPV/PC ratio was an independent predictor for CSS ( $P$ $<0.001)$. When we set the cut-off point using ROC curve, the MPV/PC ratio was still an independent predictor for CSS $(P<0.001)$.

Conclusion. The MPV/PC ratio is a useful indicator in patients with ESCC. 
1 Prognostic value of mean platelet volume/platelet count ratio in patients with resectable

2 esophageal squamous cell carcinoma: a retrospective study

3

4 Ji-Feng Feng ${ }^{1,2}$, Chen Sheng ${ }^{1}$, Qiang Zhao ${ }^{1,2}$, Peng-Cheng Chen ${ }^{1}$

5

6 Ji-Feng Feng

$7 \quad$ Email: Fengjf@zjcc.org.cn

8

9 Chen Sheng

10 Email: Chensheng@zjcc.org.cn

12 Qiang Zhao

13 Email: Zhaoqiang@zjcc.org.cn

Peng-Cheng Chen

Email: Chenpc0425@126.com

1 Department of Thoracic Surgery, Zhejiang Cancer Hospital, Hangzhou 310022, China.

2 Key Laboratory Diagnosis and Treatment Technology on Thoracic Oncology, Zhejiang province, Hangzhou 310022, China.

Corresponding author

Ji-Feng Feng Email: Fengjf@zjcc.org.cn

Peng-Cheng Chen Email: Chenpc0425@126.com 


\section{Abstract}

Background. Mean platelet volume (MPV) to platelet count (PC) ratio (MPV/PC) is a useful indicator in several cancers. However, the role for MPV/PC ratio in esophageal squamous cell carcinoma (ESCC) is still controversial.

Methods. A retrospective study was conducted including 277 resectable ESCC patients. The optimal cut-off values were calculated by the X-tile program. The receiver operator characteristic (ROC) curves were also created to show the candidate cut-off points. The comparisons between the X-tile plot and ROC curve were performed. The Kaplan-Meier method was utilized to analyze the cancer-specific survival (CSS). Prognostic factors for CSS were calculated with Cox regression univariate and multivariate analyses.

Results. According to the X-tile program, the cut-off values for MPV, PC and MPV/PC ratio were 8.5 (fl), 200 (giga/l) and 0.04, respectively. However, the cut-off values for MPV, PC and MPV/PC ratio by the ROC curves were 8.25 (fl), 243.5 (giga/l) and 0.0410, respectively. The cut-off values were similar between the X-tile and ROC curve. A low MPV/PC ratio level $(\leq 0.04)$ was associated with poor CSS $(22.4 \%$ vs. $43.1 \%, P<0.001)$. In multivariate analyses, we found that MPV/PC ratio was an independent predictor for CSS $(P<0.001)$. When we set the cut-off point using ROC curve, the MPV/PC ratio was still an independent predictor for CSS $(P<0.001)$.

Conclusion. The MPV/PC ratio is a useful predictive indicator in patients with ESCC. 
Introduction

61

Esophageal cancer (EC) is the 8th most common cancer worldwide and the 6th most common cause of death from cancer (Ferlay et al., 2010). The incidences vary widely in different countries and regions. To date, approximately $53.8 \%$ and $51.9 \%$ of all ECs occurred and died in China (Siegel et al., 2015; Ferlay et al., 2010). Esophageal adenocarcinoma is the most common malignancy in the West. In China, however, esophageal squamous cell carcinoma (ESCC) is the predominant subtype (Napier et al., 2014). Radical esophagectomy remains the most effective therapy for patients with EC. However, the prognosis for EC remains poor (Bedenne et al., 2007; Domper et al., 2015). Therefore, it is very important to find more and more useful and effective prognostic indicators for patients with EC.

Over the past few decades, a number of prognostic factors for EC have been identified, including tumor length, vessel invasion, lymph node status ( $\mathrm{N}$ stage), depth of invasion ( $\mathrm{T}$ stage), TNM stage and other serum biomarkers, such as squamus cell carcinoma antigen (SCCA) and carcinoembryonic antigen (CEA) (Peyre et al., 2008; Wijnhoven et al., 2007; Feng et al., 2013). Inflammation plays an important role in cancer progression and prognosis (Balkwill et al., 2001; Mantovani et al., 2008). C-reactive protein (CRP), as a most sensitive inflammatory biomarker, has been confirmed in a series of cancers to predict the prognosis, including patients with EC (Shimada et al., 2003; Nozoe et al., 2001; Platt et al., 2012). In addition, there are other 
parameters like neutrophil and lymphocyte that are easy-to-measure inflammatory markers (Dutta et al., 2011).

Mean platelet volume (MPV) is recognized as a hallmark for platelet count (PC) activation (Kamath et al., 2001). Several studies showed that MPV and PC are associated with mortality in cardiovascular disease, such as ischemic cardiovascular disease and acute myocardial infarction (Guenancia et al., 2017; Azab et al., 2011). Moreover, recent studies have shown that the ratio for MPV to PC (MPV/PC) is associated with prognosis in some malignancies, such as hepatocellular carcinoma and lung cancer (Cho et al., 2013; Inagaki et al., 2014; Omar et al., 2018). However, the role for MPV/PC ratio in ESCC is still controversial. Furthermore, controversy exists concerning the optimal cut-off points for MPV/PC to predict the prognosis of ESCC. Therefore, the purpose of our study here was to explore the prognostic role of MPV/PC ratio in patients with ESCC.

\section{Patients and Methods}

From January 2007 to December 2010 at the Department of Thoracic Surgery, Zhejiang Cancer Hospital, a retrospective study was conducted including 277 resectable ESCC patients. The exclusion criteria were as follows: 1). patients who received preoperative treatment, such as chemotherapy and/or radiotherapy; 2). patients who had any form of acute or chronic inflammatory diseases or infections; 3). patients who had systemic diseases, and 4) those diagnosed with distant metastases. Written informed consent for the collection of specimen and other medical information were obtained from all patients before surgery. The current study was approved by the Ethics Committees of Zhejiang Cancer Hospital (IRB Approval No. IRB-2018130).

The main clinical characteristics, such as age, gender, tumor location (upper, middle and lower), tumor length, vessel invasion, differentiation (well, moderate and poor) and tumor stage (T stage, 
106

107

108

109

110

111

112

113

114

115

116

117

118

119

120

121

122

123

124

125

126

127

128

129

130

131

132

$\mathrm{N}$ stage and TNM stage), were retrospectively reviewed and collected. The tumor length was defined as the long diameter for pathological specimens. Blood samples were obtained within one week prior to surgery to measure the neutrophil (Neu), MPV, PC, CRP and CEA levels. MPV/PC was defined as MPV to PC ratio. Neu/PC was defined as Neu to PC ratio. The levels of Neu, MPV and PC were measured by automated blood cell counter (Sysmex XE-2100, Kobe, Japan). Serum levels of CRP were determined by latex-enhanced homogeneous immunoassay (Hitachi 917; Skill, Munich, Germany). Serum levels of CEA were measured using enzyme immunoassay kits (Abbott, Chicago, USA). The AJCC/UICC TNM staging system (the 7th edition) was utilized to classify the stage for this study (Rice et al., 2010).

All the above patients were followed-up postoperatively (regularly evaluated every 3-6 months). The assessment included physical examination, blood tumor markers and computed tomography scan. In this study, we conducted a cancer-specific survival (CSS) to analyze the prognosis of patients with ESCC. The mean follow-up for patients was 45 months.

\section{Statistical analyses}

In the current study, the optimal cut-off values for Neu, MPV, PC, MPV/PC ratio, and Neu/PC ratio were calculated by the X-tile program (Camp et al., 2004). The receiver operator characteristic (ROC) curves were also created to show the candidate cut-off points. The comparisons between the X-tile plot and ROC curve were performed. The areas under the curve (AUC) for Neu, MPV, PC, MPV/PC and Neu/PC were calculated and compared by the ROC curve. The chi-squared tests were used to compare the MPV/PC ratio, MPV and PC. The CSS curves were generated by the Kaplan-Meier method. Univariate analyses were performed with log-rank test. Multivariate analyses with cox proportional hazards regression model were utilized to analyze prognostic factors for CSS. SPSS 20.0 (SPSS Inc., Chicago, IL, USA) was utilized to perform the statistical analyses. R 3.2.3 software (Institute for Statistics and Mathematics, Vienna, Austria) was also utilized to conduct the nomogram model by Harrell's concordance 
133

134

135

136

137

138

139

140

141

142

143

144

145

146

147

148

149

150

151

152

153

154

155

156

157

158

159

index (c-index) (Iasonos et al., 2008).

\section{Results}

There were $37(13.4 \%)$ women and $240(86.6 \%)$ men in all 277 patients with the mean age of $59.2 \pm 7.8$ years (36-80 years). In the current study, the mean values for Neu, MPV, PC, MPV/PC and Neu/PC were $4.2 \pm 1.5$ (giga/l) (range 1.5-9.5 giga/l), $9.3 \pm 1.3(\mathrm{fl})$ (range 6.7-12.9 fl), $232 \pm 72($ giga/l) (range 60-473 giga/l), $0.04 \pm 0.02$ (range 0.02-0.14), and $0.020 \pm 0.010$ (range 0.0053-0.0667), respectively. The histograms of the preoperative MPV/PC ratio, MPV and $\mathrm{PC}$ are shown in Figure 1.

According to the X-tile program, the optimum cut-off points for MPV, PC, MPV/PC, Neu and $\mathrm{Neu} / \mathrm{PC}$ ratio were 8.5 (fl), 200 (giga/l), 0.04, 4.2 (giga/l) and 0.02, respectively (Fig. 2). According to the optimum cut-off points of the above values, patients then were divided into 2 groups $(\mathrm{MPV} \leq 8.5 \mathrm{fl}$ and $>8.5 \mathrm{fl} ; \mathrm{PC} \leq 200 \mathrm{giga} / 1$ and $>200 \mathrm{giga} / 1 ; \mathrm{MPV} / \mathrm{PC}$ ratio $\leq 0.04$ and $>0.04)$. Clinicopathologic characters for the above values (MPV/PC ratio, MPV and PC) were shown in Table 1 . The levels of MPV/PC ratio were significantly correlated with the CRP levels $(P=0.029)$.

Kaplan-Meier analyses showed that a low MPV/PC ratio level $(\leq 0.04)$ was associated with poor CSS $(P<0.001)$. The 5-year CSS was $43.1 \%$ in patients with MPV/PC ratio $>0.04$, and $22.4 \%$ in patients with MPV/PC ratio $\leq 0.04$ (Fig. 3A). There were also significantly different for MPV (42.4\% vs. $27.0 \%, P=0.010)$ and $\mathrm{PC}(41.0 \%$ vs. $26.7 \%, P=0.009)$ (Fig. 3B-C). In multivariate analyses, we found that MPV/PC ratio was an independent predictor for CSS $(P<0.001)($ Table 2). In addition, TNM stage $(P<0.001)$, CEA $(P=0.019)$, Neu $(P=0.007)$ and CRP $(P<0.001)$ were other significant prognostic variables by multivariate analyses (Table 2).

We also created ROC curves to show the candidate cut-off points. The cut-off values for Neu, 
160

161

162

163

164

165

166

167

168

169

170

171

172

173

174

175

176

177

178

179

180

181

182

183

184

185

186

187

MPV, PC, MPV/PC, and Neu/PC ratio by the ROC curves were 4.25 (giga/l), 8.25(fl), 243.5 (giga/l), 0.0410, and 0.0213, respectively (Fig. 4). The candidate cut-off points and the area under ROC curve (AUC) are shown in Table 3. When we set the cut-off points using ROC curve, the MPV/PC ratio (42.7\% vs. $23.5 \%, P<0.001)$, MPV (51.7\% vs. $26.7 \%, P=0.001$ ), and PC (41.8\% vs. $19.3 \%, P<0.001)$ were also associated with CSS (D-F) (Fig. 3D-F). In multivariate analyses, MPV/PC ratio was still an independent predictor for CSS $(P<0.001)$ (Table 4).

Moreover, we wanted to predict the survival risk (CSS) for patients with ESCC, a nomogram model was conducted including age, gender, TNM, CEA, Neu, MPV/PC ratio and CRP for CSS (Fig. 5). From this model, the probability of survival for ESCC patients could be predicted (cindex $=0.72$ ).

\section{Discussion}

Our study demonstrated some important findings: (1) MPV/PC ratio was a strong predictor of CSS; (2) MPV/PC ratio, but not MPV or PC, was a useful predictive indicator. This study used X-tile program and ROC curves as candidate cut-off points. The comparisons between the X-tile plot and ROC curve were performed. The cut-off values were similar between the X-tile and ROC curve. Moreover, our study is also the first attempt to predict the survival risk by a nomogram model based on MPV/PC ratio.

Platelet activation has been demonstrated as a common phenomenon in some cardiovascular diseases (Guenancia et al., 2017; Azab et al., 2011). To assess the platelet activation status, MPV and PC are two main aspects. Moreover, studies have shown that MPV/PC ratio is associated with prognosis in some malignancies, such as hepatocellular carcinoma and lung cancer (Cho et al., 2013; Inagaki et al., 2014; Omar et al., 2018). Cho et al. (Cho et al., 2013) have shown that the ratio of MPV/PC levels in hepatocellular carcinoma were higher than the control group. Inagaki et al. (Inagaki et al., 2014) have revealed that MPV/PC ratio was significantly different on survival in lung cancer. However, Omar et al. (Omar et al., 2018) 
showed that increased MPV and increased PC were significant higher than the control group. In their study, however, MPV/PC was not an independent predictor in lung cancer.

MPV is an indicator of platelet activation. Shen et al. (Shen et al., 2018) demonstrated that reduced MPV is associated with worse survival outcome in EC. The role for MPV/PC ratio in ESCC patients has not yet been well evaluated. A study reported by Sun et al. showed that the levels of MPV/PC ratio in ESCC were significantly lower than the healthy group, and which were significantly correlated with the tumor length (Sun et al., 2018). In our study, however, the $\mathrm{MPV} / \mathrm{PC}$ ratio was not significantly correlated with the tumor length $(P=0.087)$. In addition, they revealed that decreased MPV and MPV/PC ratio were significantly associated with locally advanced ESCC. In our study, MPV was not a significant prognostic factor by multivariate analyses. Recently, Zhang et al. (Zhang et al., 2016) initial conducted a COP-MPV (combination of MPV and PC) model to predict the prognosis in ESCC. They revealed that COP-MPV was a useful independent predictor, but not for MPV or PC. As everyone knows, MPV and PC may be influenced by a variety of other non-cancer related conditions, the potential basis could be decreased by the MPV to PC ratio (MPV/PC). Therefore, the role of the MPV/PC ratio would be more reliable than the effect of either MPV or PC. In the current study, a low MPV/PC ratio level $(\leq 0.04)$ was associated with poor CSS $(P<0.001)$ and was confirmed by multivariate analyses $(P<0.001)$.

In previous studies, controversy exists about the optimum cut-off point for MPV/PC ratio to predict prognosis. Cho et al. (Cho et al., 2013) demonstrated that 0.0491 might be the optimum cut-off point for MPV/PC ratio in hepatocellular carcinoma according to the ROC curve. Inagaki et al. (Inagaki et al., 2014) and Omar et al. (Omar et al., 2018) also conducted the ROC curve analyses to calculate the optimum cut-off point for MPV/PC in lung cancer. They concluded that the optimum cut-off points for MPV/PC ratio were 0.40873 and 0.47424 , respectively. Recently, Camp et al. (Camp et al., 2004) initial conducted a program to explore the optimum cut-off point 
215 (X-tile plot). In our study, according to their method, 0.04 was the optimum cut-off point for $216 \mathrm{MPV} / \mathrm{PC}$ ratio. We also created ROC curves to show the candidate cut-off points. When we set 217 the cut-off point using ROC curve, the MPV/PC ratio was also associated with CSS. In 218 multivariate analyses, MPV/PC ratio was still an independent predictor for CSS.

The mechanism between MPV/PC ratio and cancer remains unknown. Inflammation and cancer

221

222

223

224

225

226

227

228

229

230

231

232

233

234

235

236

\section{7}

238

239

240

241 are closely related (Balkwill et al., 2001; Mantovani et al., 2008). As everyone knows, platelets can release a variety of cytokines, such as platelet-derived growth factor (PDGF) and vascular endothelial growth factor (VEGF), which have an important role in regulating angiogenesis (Blair et al., 2009; Borsig et al., 2008; Dineen et al., 2009). The inflammation will be inevitably caused by chemotherapy and/or radiation. Therefore, we analyze the role of MPV/PC ratio in ESCC patients without neoadjuvant chemotherapy and/or radiation.

Limitations should be acknowledged in this study. The major limitations of this study are small samples and its retrospective character. Moreover, patients who received preoperative chemotherapy and/or radiotherapy were excluded, which might have influenced the result in the current study. On the one hand, neoadjuvant treatment will have a side effect on MPV and PC. On the other hand, neoadjuvant treatment can improve cancer survival for locally advanced EC, but not for early stage EC (Rawat et al., 2013; Mariette et al., 2014). In addition, we did not set up a validation group to verify the conclusion. Thus, the results of our study are expected more large-sample trials to confirm in future.

\section{Conclusion}

In summary, we found that the ratio of $\mathrm{MPV} / \mathrm{PC}$ is a potential prognostic biomarkers in patients with ESCC.

\section{References}


242

243

244

245

246

247

248

249

250

251

252

253

254

255

256

257

Azab B, Torbey E, Singh J, Akerman M, Khoueiry G, McGinn JT, Widmann WD, Lafferty J. 2011. Mean platelet volume/platelet count ratio as a predictor of long-term mortality after non-ST-elevation myocardial infarction. Platelets 22: 557-566. doi: $10.3109 / 09537104.2011 .584086$.

Balkwill F, Mantovani A. 2001. Inflammation and cancer: back to Virchow? Lancet 357: 539545. doi: 10.1016/S0140-6736(00)04046-0.

Bedenne L, Michel P, Bouche O, Milan C, Mariette C, Conroy T, Pezet D, Roullet B, Seitz JF, Herr JP, Paillot B, Arveux P, Bonnetain F, Binquet C. 2007. Chemoradiation followed by surgery compared with chemoradiation alone in squamous cancer of the esophagus: FFCD 9102. J Clin Oncol 25: 1160-1168. doi: 10.1200/JCO.2005.04.7118.

Blair P, Flaumenhaft R. 2009. Platelet alpha-granules: basic biology and clinical correlates. Blood Rev 23: 177-189. doi: 10.1016/j.blre.2009.04.001.

Borsig L. 2008. The role of platelet activation in tumor metastasis. Expert Rev Anticancer Ther 8: 1247-1255. doi: 10.1586/14737140.8.8.1247.

Camp RL, Dolled-Filhart M, Rimm DL. 2004. X-tile: a new bio-informatics tool for biomarker assessment and outcome-based cut-point optimization. Clin Cancer Res 10: 72527259. doi: 10.1158/1078-0432.CCR-04-0713.

Cho SY, Yang JJ, You E, Kim BH, Shim J, Lee HJ, Lee WI, Suh JT, Park TS. 2013. Mean platelet volume/platelet count ratio in hepatocellular carcinoma. Platelets 24: 375-377. doi: 10.3109/09537104.2012.701028.

Dineen SP, Roland CL, Toombs JE, Kelher M, Silliman CC, Brekken RA, Barnett CC Jr. 2009. The acellular fraction of stored platelets promotes tumor cell invasion. J Surg Res 153: 132-137. doi: 10.1016/j.jss.2008.04.013.

Domper Arnal MJ, Ferrández Arenas Á, Lanas Arbeloa Á. 2015. Esophageal cancer: Risk factors, screening and endoscopic treatment in Western and Eastern countries. World $J$ Gastroenterol 21: 7933-7943. doi: 10.3748/wjg.v21.i26.7933. 
268

269

270

271

272

273

274

275

276

277

278

279

280

281

282

283

284

285

286

287

288

289

290

291

292

293

294

Dutta S, Crumley AB, Fullarton GM, Horgan PG, McMillan DC. 2011. Comparison of the prognostic value of tumour- and patient-related factors in patients undergoing potentially curative resection of oesophageal cancer. World J Surg 35: 1861-1866. doi: 10.1007/s00268011-1130-7.

Feng JF, Huang Y, Zhao Q. 2013. Tumor length in elderly patients with esophageal squamous cell carcinoma: is it a prognostic factor? Ups J Med Sci 118: 145-152. doi: 10.3109/03009734.2013.792887.

Ferlay J, Shin HR, Bray F, Forman D, Mathers C, Parkin DM. 2010. Estimates of worldwide burden of cancer in 2008: GLOBOCAN 2008. Int J Cancer 127: 2893-2917. doi: 10.1002/ijc.25516.

Guenancia C, Hachet O, Stamboul K, Béjot Y, Leclercq T, Garnier F, Yameogo NV, de Maistre E, Cottin Y, Lorgis L. 2017. Incremental predictive value of mean platelet volume to platelet count ratio in in-hospital stroke after acute myocardial infarction. Platelets 28: 5459. doi: 10.1080/09537104.2016.1203397.

Iasonos A, Schrag D, Raj GV, Panageas KS. 2008. How to build and interpret a nomogram for cancer prognosis. J Clin Oncol 26: 1364-1370. doi: 10.1200/JCO.2007.12.9791.

Inagaki N, Kibata K, Tamaki T, Shimizu T, Nomura S. 2014. Prognostic impact of the mean platelet volume/platelet count ratio in terms of survival in advanced non-small cell lung cancer. Lung Cancer 83: 97-101. doi: 10.1016/j.lungcan.2013.08.020.

Kamath S, Blann AD, Lip GY. 2001. Platelet activation: assessment and quantification. Eur Heart J 22: 1561-1571. doi: 10.1053/euhj.2000.2515.

Mariette C, Dahan L, Mornex F, Maillard E, Thomas PA, Meunier B, Boige V, Pezet D, Robb WB, Le Brun-Ly V. 2014. Surgery alone versus chemoradiotherapy followed by surgery for stage I and II esophageal cancer: final analysis of randomized controlled phase III trial FFCD 9901. J Clin Oncol 32: 2416-2422. doi: 10.1200/JCO.2013.53.6532.

Mantovani A, Allavena P, Sica A, Balkwill F. 2008. Cancer-related inflammation. Nature 454: 436-444. doi: 10.1038/nature07205. 
295

296

297

298

299

300

301

302

303

304

305

306

307

308

309

310

311

312

313

314

315

316

317

318

319

320

Napier KJ, Scheerer M, Misra S. 2014. Esophageal cancer: A review of epidemiology, pathogenesis, staging workup and treatment modalities. World J Gastrointest Oncol 6: 112120. doi: 10.4251/wjgo.v6.i5.112.

Nozoe T, Saeki H, Sugimachi K. 2001. Significance of preoperative elevation of serum Creactive protein as an indicator of prognosis in esophageal carcinoma. Am J Surg 182: 197-201.

Omar M, Tanriverdi O, Cokmert S, Oktay E, Yersal O, Pilancı KN, Menekse S, Kocar M, Sen CA, Ordu C. 2018. Role of increased mean platelet volume (MPV) and decreased MPV/platelet count ratio as poor prognostic factors in lung cancer. Clin Respir J 12: 922-929. doi: $10.1111 /$ crj.12605.

Peyre CG, Hagen JA, DeMeester SR, Altorki NK, Ancona E, Griffin SM, Hölscher A, Lerut T, Law S, Rice TW, Ruol A, van Lanschot JJ, Wong J, DeMeester TR. 2008. The number of lymph nodes removed predicts survival in esophageal cancer: an international study on the impact of extent of surgical resection. Ann Surg 248: 549-556. doi: 10.1097/SLA.0b013e318188c474.

Platt JJ, Ramanathan ML, Crosbie RA, Anderson JH, McKee RF, Horgan PG, McMillan DC. 2012. C-reactive protein as a predictor of postoperative infective complications after curative resection in patients with colorectal cancer. Ann Surg Oncol 19: 4168-4177. doi: 10.1245/s10434-012-2498-9.

Rawat S, Kumar G, Kakria A, Sharma MK, Chauhan D. 2013. Chemoradiotherapy in the management of locally advanced squamous cell carcinama esophagus: is surgical resection required? J Gastrointest Cancer 44: 277-284. doi: 10.1007/s12029-013-9477-7.

Rice TW, Rusch VW, Ishwaran H, Blackstone EH. 2010. Cancer of the esophagus and esophagogastric junction: data-driven staging for the seventh edition of the American Joint Committee on Cancer/International Union Against Cancer Staging Manuals. Cancer 116: 3763-3773. doi: 10.1002/cncr.25146.

Shen W, Cui MM, Wang X, Wang RT. 2018. Reduced mean platelet volume is associated with 
321 poor prognosis in esophageal cancer. Cancer Biomark 22: 559-563. doi: 10.3233/CBM$322 \quad 181231$.

323 Shimada H, Nabeya Y, Okazumi S, Matsubara H, Shiratori T, Aoki T, Sugaya M, 324 Miyazawa Y, Hayashi H, Miyazaki S, Ochiai T. 2003. Elevation of preoperative serum Creactive protein level is related to poor prognosis in esophageal squamous cell carcinoma. $J$ Surg Oncol 83: 248-252. doi: 10.1002/jso.10275.

Siegel RL, Miller KD, Jemal A. 2015. Cancer statistics, 2015. CA Cancer J Clin 65: 5-29. doi: $10.3322 /$ caac. 21254 .

Sun SY, Zhao BQ, Wang J, Mo ZX, Zhao YN, Wang Y, He J. 2018. The clinical implications of mean platelet volume and mean platelet volume/platelet count ratio in locally advanced esophageal squamous cell carcinoma. Dis Esophagus 31: 2. doi: 10.1093/dote/dox125.

Wijnhoven BP, Tran KT, Esterman A, Watson DI, Tilanus HW. 2007. An evaluation of prognostic factors and tumor staging of resected carcinoma of the esophagus. Ann Surg 245: 717-725. doi: 10.1097/01.sla.0000251703.35919.02.

Zhang F, Chen Z, Wang P, Hu X, Gao Y, He J. 2016. Combination of platelet count and mean platelet volume (COP-MPV) predicts postoperative prognosis in both resectable early and advanced stage esophageal squamous cell cancer patients. Tumour Biol 37: 9323-9331. doi: 10.1007/s13277-015-4774-3. 


\section{Table $\mathbf{1}$ (on next page)}

Comparison of baseline clinical characteristics in ESCC

Comparison of baseline clinical characteristics in ESCC 
Table 1 Comparison of baseline clinical characteristics in ESCC

\begin{tabular}{|c|c|c|c|c|c|c|c|c|c|c|}
\hline & \multirow{2}{*}{ Total } & \multicolumn{2}{|c|}{ MPV (fl) } & \multirow[t]{2}{*}{$P$ value } & \multicolumn{2}{|c|}{ PC (giga/l) } & \multirow[t]{2}{*}{$P$ value } & \multicolumn{2}{|c|}{$\mathrm{MPV} / \mathrm{PC}$} & \multirow[t]{2}{*}{$P$ value } \\
\hline & & $\leq 8.5$ & $>8.5$ & & $\overline{\leq 200}$ & $>200$ & & $\leq 0.04$ & $>0.04$ & \\
\hline Age (years) & & & & 0.704 & & & 0.221 & & & 0.488 \\
\hline$\leq 60$ & 158 & 51 & 107 & & 55 & 103 & & 81 & 77 & \\
\hline$>60$ & 119 & 41 & 78 & & 50 & 69 & & 66 & 53 & \\
\hline Gender & & & & 0.521 & & & 0.271 & & & 0.629 \\
\hline Female & 37 & 14 & 23 & & 11 & 26 & & 21 & 16 & \\
\hline Male & 240 & 78 & 162 & & 94 & 146 & & 126 & 114 & \\
\hline Tumor length $(\mathrm{cm})$ & & & & 0.246 & & & 0.020 & & & 0.087 \\
\hline$\leq 3.0$ & 78 & 30 & 48 & & 38 & 40 & & 35 & 43 & \\
\hline$>3.0$ & 199 & 62 & 137 & & 67 & 132 & & 112 & 87 & \\
\hline CRP (mg/l) & & & & 0.031 & & & 0.152 & & & 0.029 \\
\hline$\leq 10.0$ & 200 & 74 & 126 & & 81 & 119 & & 98 & 102 & \\
\hline$>10.0$ & 77 & 18 & 59 & & 24 & 53 & & 49 & 28 & \\
\hline Tumor location & & & & 0.242 & & & 0.096 & & & 0.057 \\
\hline Upper & 16 & 7 & 9 & & 10 & 6 & & 4 & 12 & \\
\hline Middle & 127 & 36 & 91 & & 44 & 83 & & 72 & 55 & \\
\hline Lower & 134 & 49 & 85 & & 51 & 83 & & 71 & 63 & \\
\hline Vessel invasion & & & & 0.744 & & & 0.097 & & & 0.111 \\
\hline Negative & 232 & 78 & 154 & & 83 & 149 & & 128 & 104 & \\
\hline Positive & 45 & 14 & 31 & & 22 & 23 & & 19 & 26 & \\
\hline Differentiation & & & & 0.927 & & & 0.826 & & & 0.454 \\
\hline Well & 43 & 15 & 28 & & 16 & 27 & & 25 & 18 & \\
\hline Moderate & 179 & 58 & 121 & & 70 & 109 & & 90 & 89 & \\
\hline Poor & 55 & 19 & 36 & & 19 & 36 & & 32 & 23 & \\
\hline T stage & & & & 0.106 & & & 0.313 & & & 0.425 \\
\hline $\mathrm{T} 1$ & 50 & 22 & 28 & & 18 & 32 & & 28 & 22 & \\
\hline $\mathrm{T} 2$ & 49 & 20 & 29 & & 24 & 25 & & 21 & 28 & \\
\hline $\mathrm{T} 3$ & 154 & 44 & 110 & & 56 & 98 & & 86 & 68 & \\
\hline $\mathrm{T} 4$ & 24 & 6 & 18 & & 7 & 17 & & 12 & 12 & \\
\hline $\mathrm{N}$ stage & & & & 0.054 & & & 0.720 & & & 0.899 \\
\hline N0 & 150 & 60 & 90 & & 61 & 89 & & 78 & 72 & \\
\hline N1 & 74 & 21 & 53 & & 27 & 47 & & 39 & 35 & \\
\hline $\mathrm{N} 2$ & 32 & 7 & 25 & & 10 & 22 & & 19 & 13 & \\
\hline N3 & 21 & 4 & 17 & & 7 & 14 & & 11 & 10 & \\
\hline TNM stage & & & & 0.003 & & & 0.357 & & & 0.546 \\
\hline I & 69 & 31 & 38 & & 31 & 38 & & 33 & 36 & \\
\hline II & 92 & 35 & 57 & & 34 & 58 & & 52 & 40 & \\
\hline III & 116 & 26 & 90 & & 40 & 76 & & 62 & 54 & \\
\hline CEA (ng/ml) & & & & 0.818 & & & 0.566 & & & 0.954 \\
\hline
\end{tabular}




\begin{tabular}{lllllllllll}
\hline$\leq 5.0$ & 239 & 80 & 159 & & 89 & 50 & & 127 & 112 & \\
$>5.0$ & 38 & 12 & 26 & & 16 & 22 & & 20 & 18 & \\
$\quad$ Neu (giga/l) & & & & 0.249 & & & 0.681 & & & 0.186 \\
$\quad \leq 4.2$ & 146 & 53 & 93 & & 57 & 89 & & 72 & 74 & \\
$>4.2$ & 131 & 39 & 92 & & 48 & 83 & & 75 & 56 & \\
Neu/PC & & & & 0.090 & & & $<0.001$ & & & $<0.001$ \\
$\leq 0.02$ & 170 & 50 & 120 & & 35 & 135 & & 114 & 56 & \\
$>0.02$ & 107 & 42 & 65 & & 70 & 37 & & 33 & 74 & \\
\hline
\end{tabular}

2 ESCC: esophageal squamous cell carcinoma; CRP: c-reactive protein; MPV: mean platelet volume; PC:

3 platelet count; TNM: tumor node metastasis; CEA: carcinoembryonic antigen; Neu: neutrophil. 


\section{Table 2 (on next page)}

Univariate and multivariate analyses for cancer-specific survival

Univariate and multivariate analyses for cancer-specific survival 
1 Table 2 Univariate and multivariate analyses for cancer-specific survival

\begin{tabular}{|c|c|c|c|c|c|c|}
\hline & CSS $(\%)$ & $P$ value & $\frac{\text { Univariate analysis }}{\mathrm{HR}(95 \% \mathrm{CI})}$ & $P$ value & $\frac{\text { Multivariate analysis }}{\mathrm{HR}(95 \% \mathrm{CI})}$ & $\mathrm{P}$ value \\
\hline Age (years) & & 0.412 & & 0.417 & - & - \\
\hline$\leq 60$ & 33.5 & & 1.000 & & & \\
\hline$>60$ & 30.3 & & $1.127(0.845-1.502)$ & & & \\
\hline Gender & & 0.114 & & 0.120 & - & - \\
\hline Female & 45.9 & & 1.000 & & & \\
\hline Male & 30.0 & & $1.445(0.909-2.298)$ & & & \\
\hline Tumor length & & 0.003 & & 0.004 & - & - \\
\hline$\leq 3 \mathrm{~cm}$ & 42.3 & & 1.000 & & & \\
\hline$>3 \mathrm{~cm}$ & 28.1 & & $1.642(1.173-2.297)$ & & & \\
\hline Tumor location & & 0.336 & & 0.342 & - & - \\
\hline Upper/Middle & 35.7 & & 1.000 & & & \\
\hline Lower & 28.4 & & $1.149(0.863-1.530)$ & & & \\
\hline Vessel invasion & & 0.003 & & 0.003 & - & - \\
\hline Negative & 35.3 & & 1.000 & & & \\
\hline Positive & 15.6 & & $1.710(1.197-2.444)$ & & & \\
\hline Differentiation & & 0.054 & & 0.058 & - & - \\
\hline Well/Moderate & 33.8 & & 1.000 & & & \\
\hline Poor & 25.5 & & $1.398(0.989-1.978)$ & & & \\
\hline T stage & & $<0.001$ & & $<0.001$ & - & - \\
\hline $\mathrm{T} 1-2$ & 45.5 & & 1.000 & & & \\
\hline T3-4 & 24.7 & & $1.898(1.382-2.606)$ & & & \\
\hline $\mathrm{N}$ stage & & $<0.001$ & & $<0.001$ & - & - \\
\hline No & 49.3 & & 1.000 & & & \\
\hline N1-3 & 11.8 & & $2.852(2.120-3.836)$ & & & \\
\hline TNM stage & & $<0.001$ & & $<0.001$ & & $<0.001$ \\
\hline $\mathrm{I}$ & 58.0 & & 1.000 & & 1.000 & \\
\hline II & 38.9 & & $1.966(1.259-3.067)$ & 0.003 & $1.825(1.164-2.861)$ & 0.009 \\
\hline III & 13.8 & & $3.799(2.490-5.736)$ & $<0.001$ & $3.624(2.362-5.560)$ & $<0.001$ \\
\hline Adjuvant therapy & & 0.085 & & 0.090 & - & - \\
\hline No & 35.6 & & 1.000 & & & \\
\hline Yes & 24.4 & & $1.297(0.960-1.753)$ & & & \\
\hline $\mathrm{CRP}(\mathrm{mg} / \mathrm{l})$ & & $<0.001$ & & $<0.001$ & & $<0.001$ \\
\hline$\leq 10.0$ & 39.5 & & 1.000 & & 1.000 & \\
\hline$>10.0$ & 13.0 & & $2.066(1.526-2.798)$ & & $1.994(1.461-2.722)$ & \\
\hline MPV (fl) & & 0.019 & & 0.021 & - & - \\
\hline$\leq 8.5$ & 41.3 & & 1.000 & & & \\
\hline$>8.5$ & 27.6 & & $1.451(1.057-1.992)$ & & & \\
\hline PC (giga/l) & & 0.009 & & 0.011 & - & - \\
\hline
\end{tabular}




\begin{tabular}{|c|c|c|c|c|c|c|}
\hline$\leq 200$ & 41.0 & & 1.000 & & & \\
\hline$>200$ & 26.7 & & $1.488(1.097-2.019)$ & & & \\
\hline MPV/PC & & $<0.001$ & & $<0.001$ & & $<0.001$ \\
\hline$>0.04$ & 43.1 & & 1.000 & & 1.000 & \\
\hline$\leq 0.04$ & 22.4 & & $1.861(1.386-2.498)$ & & $1.823(1.347-2.469)$ & \\
\hline $\mathrm{CEA}(\mathrm{ng} / \mathrm{ml})$ & & 0.027 & & 0.031 & & 0.019 \\
\hline$\leq 5.0$ & 33.5 & & 1.000 & & 1.000 & \\
\hline$>5.0$ & 23.7 & & $1.549(1.042-2.302)$ & & $1.613(1.082-2.407)$ & \\
\hline Neu (giga/l) & & $<0.001$ & & $<0.001$ & & 0.007 \\
\hline$\leq 4.2$ & 43.8 & & 1.000 & & 1.000 & \\
\hline$>4.2$ & 19.1 & & $1.945(1.455-2.600)$ & & $1.512(1.120-2.040)$ & \\
\hline $\mathrm{Neu} / \mathrm{PC}$ & & 0.223 & & 0.229 & - & - \\
\hline$\leq 0.02$ & 35.3 & & 1.000 & & & \\
\hline$>0.02$ & 27.1 & & $1.195(0.894-1.597)$ & & & \\
\hline
\end{tabular}

2 ESCC: esophageal squamous cell carcinoma; CRP: c-reactive protein; MPV: mean platelet volume; PC:

3 platelet count; TNM: tumor node metastasis; CEA: carcinoembryonic antigen; Neu: neutrophil; CI: confidence 4 interval; HR: hazard ratio. 


\section{Table 3 (on next page)}

Comparison of AUC areas for the prognostic factors in ESCC

Comparison of AUC areas for the prognostic factors in ESCC 
1 Table 3 Comparison of AUC areas for the prognostic factors in ESCC

\begin{tabular}{llccccc}
\hline & Cut-off & Sensibility & Specificity & AUC & $95 \%$ CI & P-value \\
\hline MPV/PC & 0.0410 & 62.9 & 61.7 & 0.608 & $0.548-0.666$ & Reference \\
MPV & 8.25 & 84.6 & 34.8 & 0.609 & $0.549-0.667$ & 0.9834 \\
PC & 243.5 & 50.0 & 74.2 & 0.648 & $0.588-0.704$ & 0.0181 \\
Neu & 4.25 & 53.7 & 76.4 & 0.689 & $0.630-0.743$ & 0.1123 \\
Neu/PC & 0.0213 & 38.3 & 74.2 & 0.543 & $0.482-0.603$ & 0.3269 \\
\hline
\end{tabular}

2 ESCC: esophageal squamous cell carcinoma; AUC: area under the curve; MPV: mean platelet volume; PC:

3 platelet count; Neu: neutrophil. 


\section{Table 4 (on next page)}

Multivariate analyses with the cut-off values by ROC curve

Multivariate analyses in ESCC with the cut-off values by ROC curve 
1 Table 4 Multivariate analyses in ESCC with the cut-off values by ROC curve

\begin{tabular}{lcc} 
& HR $(95 \% \mathrm{CI})$ & P-value \\
\hline CRP $(\mathrm{mg} / \mathrm{l})(>10.0$ vs. $\leq 10.0)$ & $2.060(1.511-2.807)$ & $<0.001$ \\
TNM stage & & \\
II vs. I & $1.816(1.160-2.844)$ & 0.009 \\
III vs. I & $3.529(2.298-5.417)$ & $<0.001$ \\
MPV/PC $(\leq 0.0410$ vs. $>0.0410)$ & $1.728(1.275-2.342)$ & $<0.001$ \\
CEA $(\mathrm{ng} / \mathrm{ml})(>5.0$ vs. $\leq 5.0)$ & $1.636(1.097-2.438)$ & 0.016 \\
Neu $($ giga/l) $(>4.25$ vs. $\leq 4.25)$ & $1.553(1.150-2.096)$ & 0.004 \\
\hline
\end{tabular}

2 ESCC: esophageal squamous cell carcinoma; CSS: cancer-specific survival; CRP: c-reactive protein; MPV:

3 mean platelet volume; PC: platelet count; TNM: tumor node metastasis; CEA: carcinoembryonic antigen; Neu:

4 neutrophil; CI: confidence interval; HR: hazard ratio.

5

6 
Figure 1

The histograms of the MPV (A), PC (B) and MPV/PC ratio (C)

The histograms of the MPV (A), PC (B) and MPV/PC ratio (C)
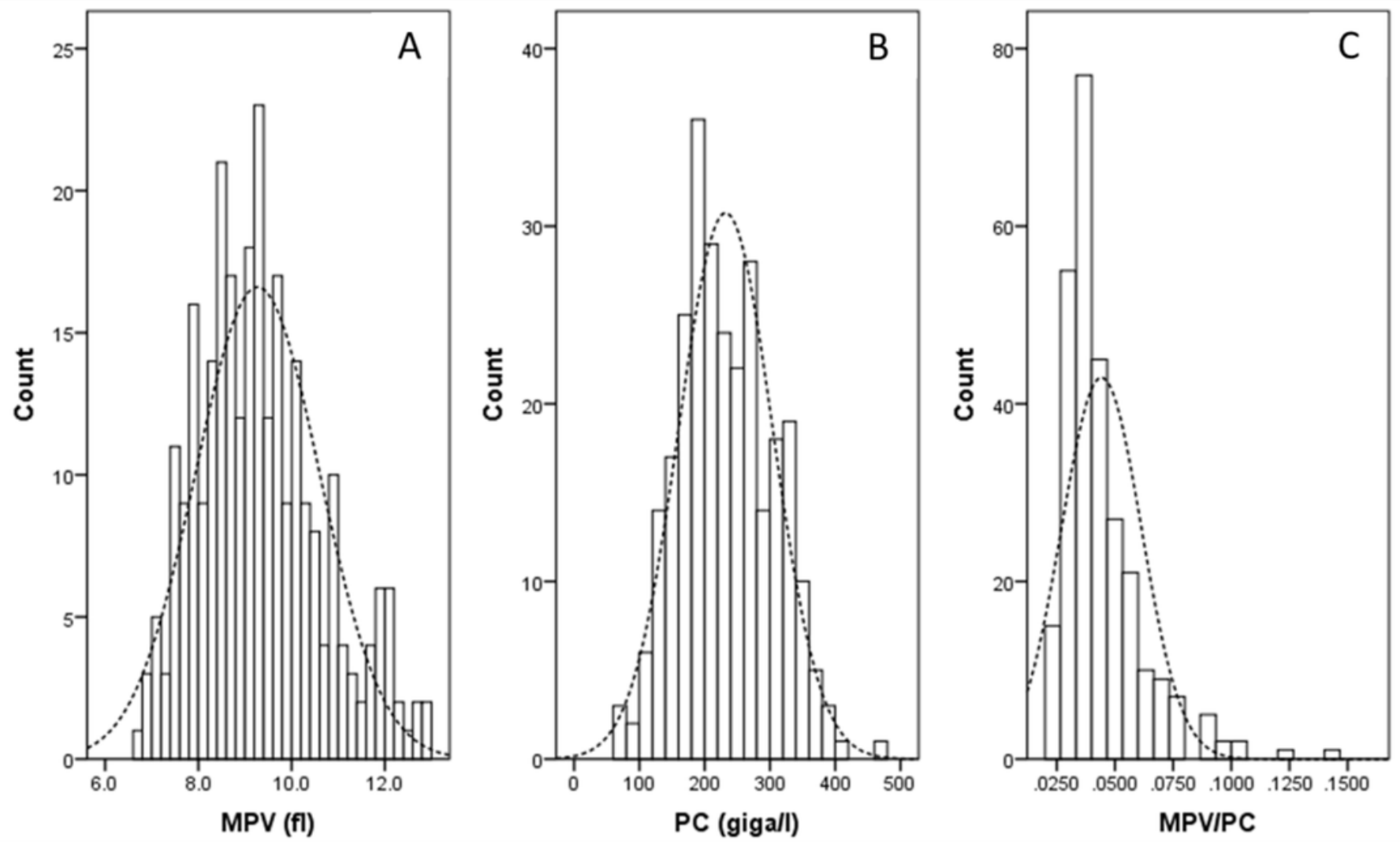


\section{Figure 2}

X-tile analyses.

X-tile plots of the training sets are shown in the left panels, with plots of matched validation sets shown in the smaller inset. The optimal cut-off points highlighted by the black circle in Fig. $A, D, G, J, M$ are shown on the histograms of the entire cohort (Fig. $B, E, H, K, N$ ) and Kaplan-Meier plots (Fig. C, F, I, L, O). According to the X-tile program, the optimum cut-off points for MPV (A-C), PC (D-F), MPV/PC (G-I), Neu (J-L) and Neu/PC ratio (M-O) were 8.5 (fl), 200 (giga/l), 0.04, 4.2 (giga/l) and 0.02, respectively. 


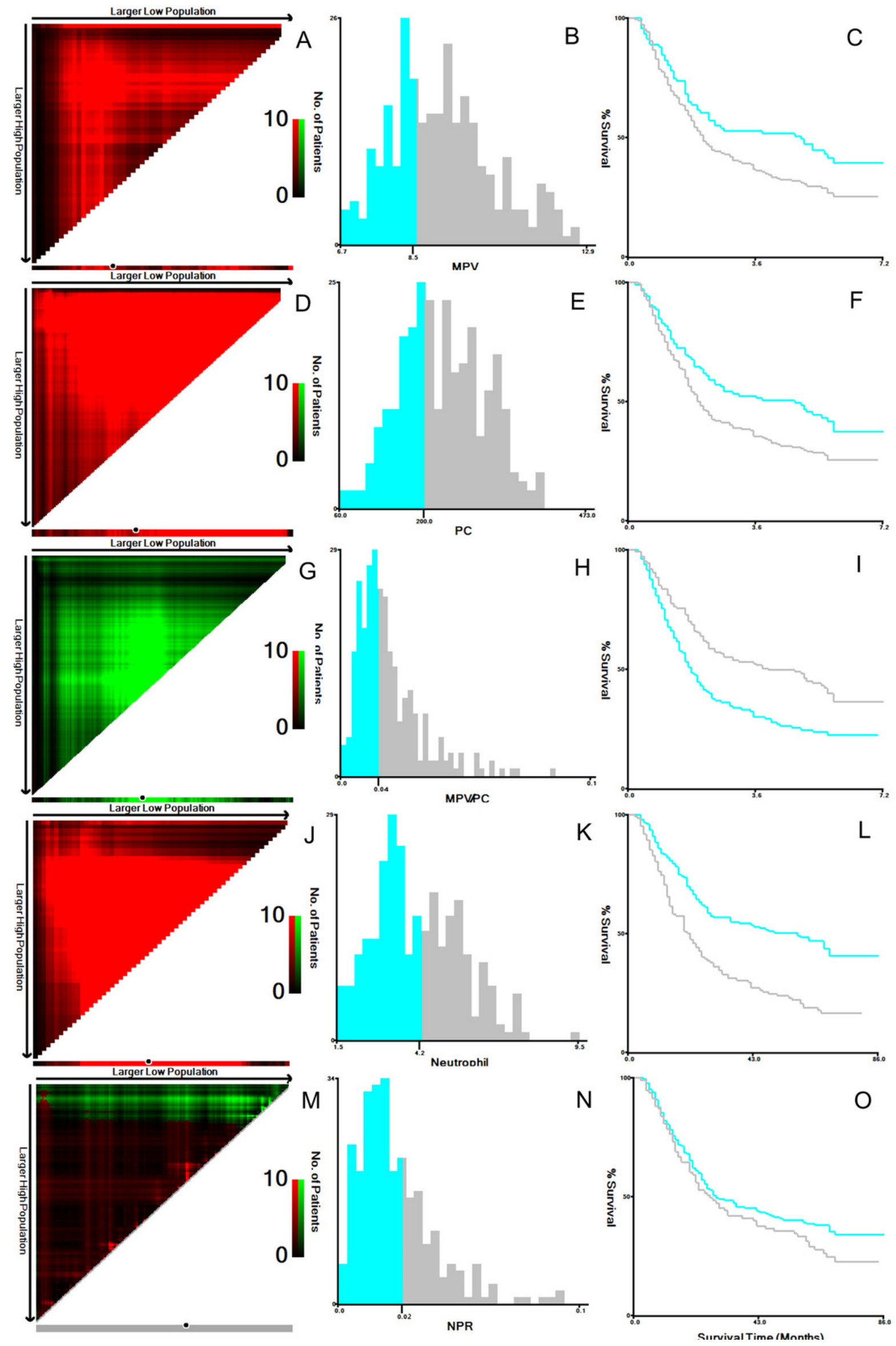

Peer) reviewing PDF | (2019:01:34245:1:1:NEW 19 May 2019) 
Figure 3

Kaplan-Meier CSS curves.

Patients with MPV/PC ratio $>0.04$ had a significantly better 5 -year CSS than patients with MPV/PC ratio $\leq 0.04$ (43.1\% vs. 22.4\%, $P<0.001 ; A)$. The 5-year CSS were also significantly different for MPV $(42.4 \%$ vs. $27.0 \%, P=0.010 ; B)$ and PC (41.0\% vs. $26.7 \%, P=0.009 ; C)$. When we set the cut-off points using ROC curve, the MPV/PC ratio $(42.7 \%$ vs. $23.5 \%, P$ $<0.001 ; \mathrm{D}), \mathrm{MPV}(51.7 \%$ vs. $26.7 \%, P=0.001 ; \mathrm{E})$, and PC (41.8\% vs. $19.3 \%, P<0.001 ; \mathrm{F})$ were also associated with CSS.
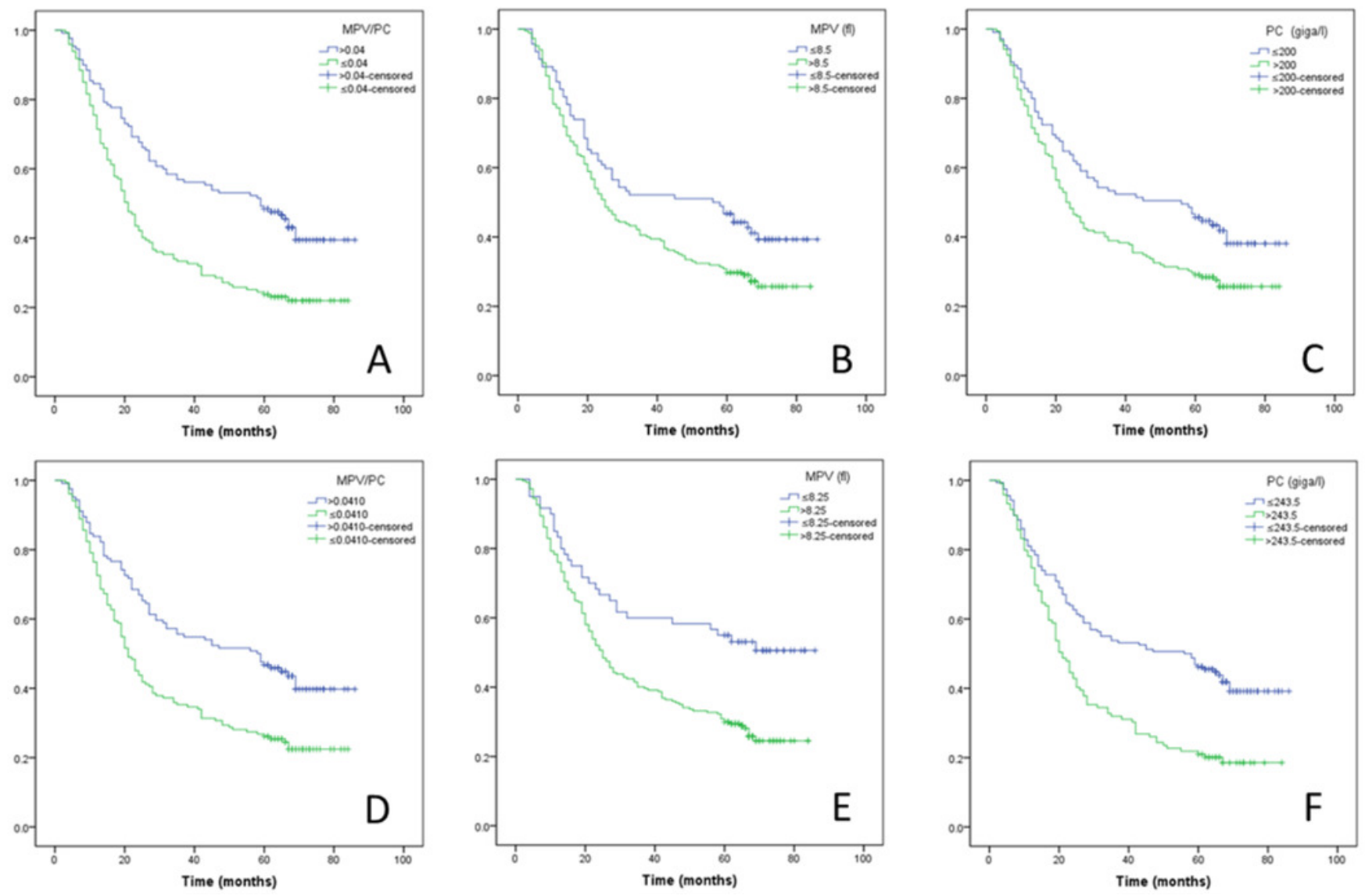
Figure 4

ROC curve analysis.

The cut-off values for Neu, MPV, PC, MPV/PC, and Neu/PC ratio by the ROC curves were 4.25 (giga/l), 8.25(fl), 243.5 (giga/l), 0.0410, and 0.0213, respectively.

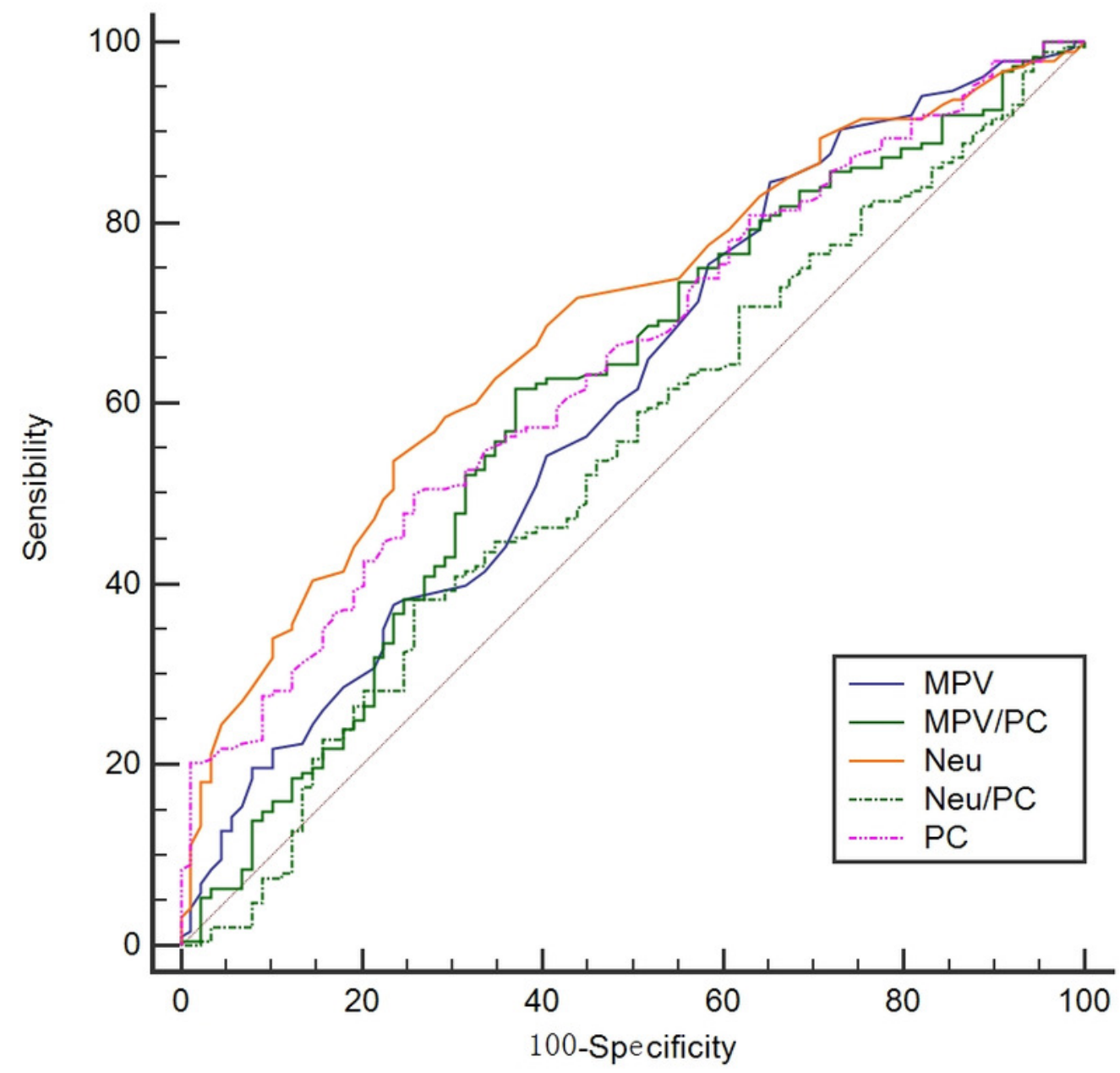




\section{Figure 5}

Nomogram model for prediction

The Harrell's c-index for CSS prediction was 0.72. A nomogram predicts survival prediction based on MPV/PC and other prognostic factors in patients with ESCC. The nomogram is used by totalling the points identified at the top of the scale for each independent factor. This total point score is then identified on the total points scale to determine the probability of risk prediction (A) and survival prediction (B). 
Points

age

gender

TNM.stage

CEA

Neu

NPR

CRP

Total Points

Risk

B

age

TNM.stage

CEA

Neu

NPR

CRP

Total Points

Linear Predictor

1-year Survival Probability

3-year Survival Probability

5-year Survival Probability

A

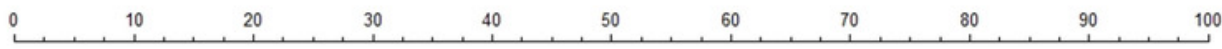
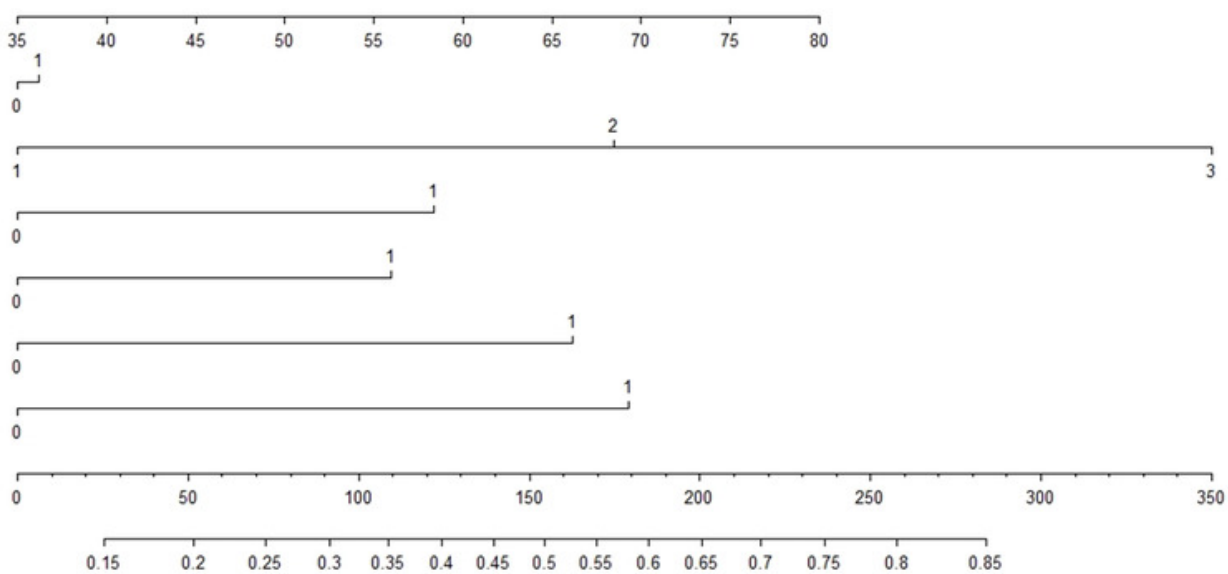
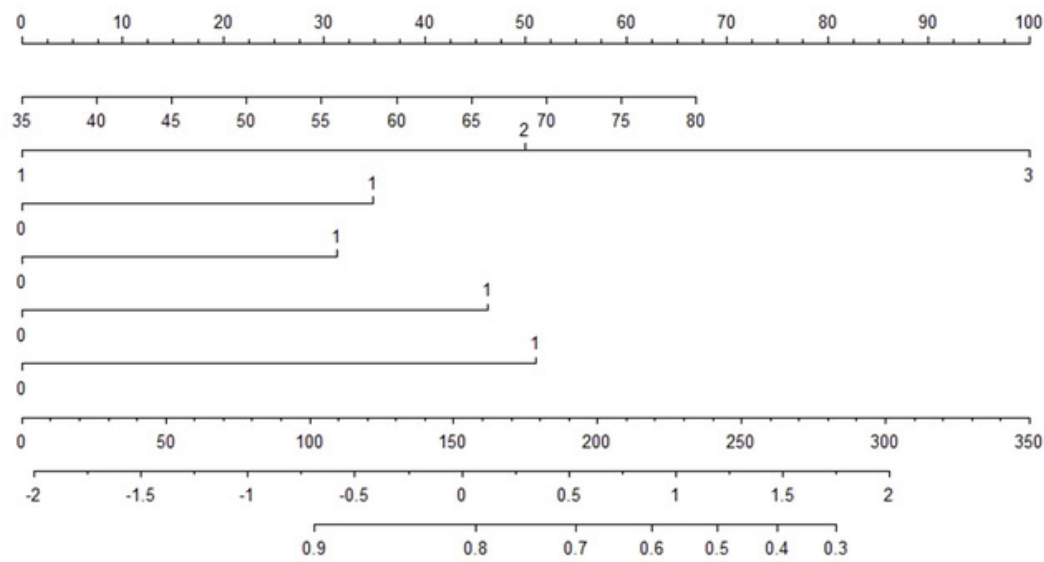

0.9

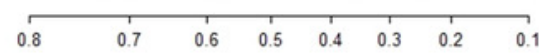

